\title{
$m$ 值随机变量序列一类极限定理的信息条件
}

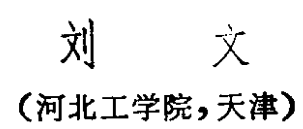

关链词相对摘密度、摘密度偏差、极限定理

设 $\left\{X_{n}, n \geqslant 1\right\}$ 是在 $S=\{1,2, \cdots, m\}$ 中取值的随机变量序列, 其联合分布为

$$
\begin{gathered}
P\left(X_{1}=x_{1}, \cdots, X_{n}=x_{n}\right)=p\left(x_{1}, \cdots, x_{n}\right)>0, \\
x_{i} \in S, 1 \leqslant i \leqslant n .
\end{gathered}
$$

令

$$
f_{n}(\omega)=-\frac{1}{n} \log p\left(X_{1}, \cdots, X_{n}\right),
$$

其中 $\log$ 是自然对数. $f_{n}(\omega)$ 称为 $\left\{X_{n}, n \geqslant 1\right\}$ 在时刻 $n$ 的相对嫡密度 ${ }^{[13}$.

$\left\{f_{n}, n \geqslant 1\right\}$ 的极限性质是信息论中的一个重要问题. 这个问题曾被很多作者研究过 ${ }^{[-s]}$. 本文的目的是要讨论这个问题和极良定理的联系, 即利用 $\left\{f_{*}\right\}$ 的极限性质给出关于 $\left\{X_{.}\right\}$的 一类极限定理的条件.

设 $\left\{X_{n}, n \geqslant 1\right\}$ 是具有分布 (1) 的一列随机变量, 则 $\left\{X_{n}, n \geqslant 1\right\}$ 相互独立的充要条 件是存在 $S$ 上的一列分布

$$
\begin{aligned}
& \alpha_{k}=\left(p_{k_{1}}, p_{k 2}, \cdots, p_{k m}\right), k=1,2, \cdots, \\
& \sum_{k=1}^{m} p_{k i}=1, p_{k i}>0,
\end{aligned}
$$

使得

$$
p\left(x_{1}, \cdots, x_{n}\right)=\prod_{i=1}^{n} p_{i x_{i}},
$$

且此时有

$$
\begin{aligned}
& P\left(X_{n}=i\right)=p_{n i}, \\
& f_{n}(\omega)=-\frac{1}{n} \sum_{i=1}^{n} \log p_{i X_{i}}, n=1,2, \cdots .
\end{aligned}
$$

定义 设 $\left\{X_{n}, n \geqslant 1\right\}$ 是具有分布 (1) 的一列随机变量, $\alpha_{k}(k=1,2, \cdots)$ 是由 (3) 与 (4) 式给定的 $S$ 上的一列分布. 令

$$
\varphi_{n}(\omega)=\frac{1}{n} \sum_{i=1}^{n} \log p_{i X_{i}}-\frac{1}{n} \log p\left(X_{1}, \cdots, X_{n}\right),
$$

$\varphi_{\text {. }}(\omega)$ 称为 $\left\{X_{i}, 1 \leqslant i \leqslant n\right\}$ 相对于独立型分布 $(5)$ 的摘密度偏差.

定理设 $\left\{X_{n}, n \geqslant 1\right\}$ 是具有分布 (1) 的一列随机变量, $k \in S, S_{n}(k, \omega)$ 是 $X_{1}(\omega)$,

本文1988 年5月11 日收到。 
$X_{2}(\omega), \cdots, X_{n}(\omega)$ 中 $k$ 出现的次数, $\varphi_{n}(\omega)$ 由 (6) 式定义, $c$ 为非负常数. 令 $D(c)=\{\omega$ : $\left.\liminf _{n \rightarrow \infty} \varphi_{*}(\omega) \geqslant-c\right\}$, 则

$$
\limsup _{n \rightarrow \infty} \frac{1}{n}\left[S_{n}(k, \omega)-\sum_{i=1}^{n} p_{i k}\right] \leqslant c+2 \sqrt{c} \quad \text { a.e., } \omega \in D(c) ;
$$

当 $0 \leqslant c<1$ 时

$$
\liminf _{n \rightarrow \infty} \frac{1}{n}\left[S_{n}(k, \omega)-\sum_{i=1}^{\infty} p_{i k}\right] \geqslant-2 \sqrt{c} \text { a.e., } \omega \in D(c) .
$$

证 取 $Q=[0,1)$, 其中的 Lebesgue 可测集的全体 和 Lebesgue 测度 $P$ 为所考 虑的概率空间. 首先给出具有分布 (1) 的随机变曋序列 $\left\{X_{n}, n \geqslant 1\right\}$ 在此概率空间中的一 种实现.

将区间 $[0,1)$ 按比例 $p(1): p(2): \cdots: p(m)$ 分成 $m$ 个左闭右开区间:

$$
\delta_{1}-[0, p(1)), \delta_{2}=[p(1), p(1)+p(2)), \cdots, \delta_{m}=[1-p(m), 1) .
$$

这些区间都称为 1 阶区间. 设 $m^{n}$ 个 $n$ 阶区间 $\left\{\delta_{x_{1} \cdots x_{n}}, x_{i}=1,2, \cdots, m, 1 \leqslant i \leqslant n\right\}$ 已经 定义. 将 $\delta_{s_{i} \cdots x_{n}}$ 按比例

$$
p\left(x_{1}, \cdots, x_{n}, 1\right): p\left(x_{1}, \cdots, x_{n}, 2\right): \cdots: p\left(x_{1}, \cdots, x_{n}, m\right)
$$

分成 $m$ 个左闭右开区间 $\delta_{x_{1} \cdots x_{n}}, \delta_{x_{1} \cdots x_{n^{2}}}, \cdots, \delta_{x_{1} \cdots x_{n} m}$, 这样就得到 $n+1$ 阶区间. 对 $n \geqslant 1$ 定义随机变量 $X_{n}:[0,1) \rightarrow S$ 如下:

$$
X_{n}(\omega)=x_{*} \text {, 当 } \omega \in \delta_{x_{1} \cdots x_{n} \bullet}
$$

易知 $P\left(\delta_{x_{1} \cdots x_{n}}\right)=p\left(x_{1}, \cdots, x_{n}\right)$, 于是 $\left\{X_{n}, n \geqslant 1\right\}$ 具有分布 (1).

设各阶区间的全体(包括零阶区间 $[0,1)$ ) 为 $\propto ⿻ 上 丨>$ 为常数. 在 $\propto$ 上定义集函数 $\mu$ 如下: 令 $\mu([0,1))-1$. 设 $\delta_{x_{1} \cdots x_{n}}$ 是 $n$ 阶区间, $k \in S, S_{n}(k)$ 是 $x_{1}, x_{2}, \cdots, x_{n}$ 中 $k$ 的个 数. 令

$$
\mu\left(\delta_{x_{1} \cdots x_{n}}\right)=\lambda^{s_{n}(k)} \prod_{i=1}^{\infty} \frac{p_{i x i}}{1+(\lambda-1) p_{i k}},
$$

易知 $\boldsymbol{\mu}$ 是 $\propto$ 上的可加集函数. 由此知存在 $[0,1)$ 上的增函数 $f_{2}$, 使得对于任何 $\delta_{x_{1}} \cdots x_{2}$ 有

$$
\mu\left(\delta_{x_{1} \cdots x_{n}}\right)=f_{2}\left(\delta_{s}^{+} \cdots x_{n}\right)-f_{2}\left(\delta_{s, \ldots x_{n}}^{-}\right),
$$

其中 $\delta_{*}, \ldots x_{n}$ 与 $\delta_{x_{1}}^{+} \ldots x_{n}$ 分别表示 $\delta_{x_{1} \cdots x_{n}}$ 的左、右端点. 令

$$
t_{n}(\lambda, \omega)=\frac{\mu\left(\delta_{x_{1} \cdots x_{n}}\right)}{P_{x}\left(\delta_{1} \cdots x_{n}\right)}=\frac{f_{2}\left(\delta_{s_{1} \cdots x_{n}}^{+}\right)-f_{2}\left(\delta_{s_{1} \cdots x_{n}}^{-}\right)}{\delta_{s_{1} \cdots x_{n}}^{+}-\delta_{s_{1} \cdots x_{n}}^{-}}, \omega \in \delta_{s_{1} \cdots x_{n}} \cdot
$$

设 $f_{2}$ 的可微点的全体为 $A_{k}(\lambda)$, 则(参见文献 [9]，p. 345)

$$
\lim _{n \rightarrow \infty} t_{n}(\lambda, \omega)=\text { 有限数, } \omega \in A_{k}(\lambda) \text {. }
$$

又由单调函数导数存在定理有 $P\left(A_{k}(\lambda)\right)=1$. 令

$$
\gamma_{n}(\omega)=\frac{\prod_{i=1}^{n} p_{i x_{i}}}{P\left(\delta_{x_{1} \cdots x_{n}}\right)}, \omega \in \delta_{x_{1} \cdots x_{n}},
$$

即

$$
r_{n}(\omega)=\frac{\prod_{i=1}^{n} p_{i X_{i}}}{P\left(X_{1}, \cdots, X_{*}\right)}, \omega \in[0,1)
$$


由 (6) 与 (14) 式有

$$
\begin{aligned}
& \varphi(\omega)=\log \left[\gamma_{n}(\omega)\right]^{1 / n}, \\
& D(c)=\left\{\omega: \liminf _{n \rightarrow \infty}\left[\gamma_{n}(\omega)\right]^{1 / n} \geqslant e^{-c}\right\} .
\end{aligned}
$$

由(13)、(11) 与 (10) 式有

$$
t_{n}(\lambda, \omega)=r_{n}(\omega) \lambda^{s_{n}^{(k)}} \prod_{i=1}^{n} \frac{1}{1+(\lambda-1) p_{i k}}, \omega \in \delta_{x_{i} \cdots x_{n}},
$$

即

$$
t_{n}(\lambda, \omega)=r_{n}(\omega) \lambda^{s_{n}(k, \omega)} \prod_{i=1}^{n} \frac{1}{1+(\lambda-1) p_{i k}}, \omega \in(0,1) .
$$

由 (12) 与 (18) 式有

$$
\limsup _{n \rightarrow \infty}\left[r_{*}(\omega)\right]^{1 / n} \lambda_{n}^{s_{n}(k, \omega) / n}\left[\prod_{n=1}^{n} \frac{1}{1+(\lambda-1) p_{i k}}\right]^{1 / n} \leqslant 1, \omega \in A_{k}(\lambda) .
$$

由 (19) 与 (16) 式有

$$
\begin{aligned}
& \left.\limsup _{n \rightarrow \infty} \lambda^{s_{u}(k, \omega) / n} \mid \prod_{i=1}^{n} \frac{1}{1+(\lambda-1)}-\right]_{i k}^{1 / n} \leqslant \frac{1}{\liminf _{n \rightarrow \infty}\left[\gamma_{n}(\omega)\right]^{1 / n}} \\
& \leqslant e^{c}, \omega \in A_{k}(\lambda) \cap D(c) .
\end{aligned}
$$

由 (20) 式有

$$
\limsup _{n \rightarrow \infty}\left[\frac{S_{k}(k, \omega)}{n} \log \lambda-\frac{1}{n} \sum_{i=1}^{\infty} \log \left(1+(\lambda-1) p_{i k}\right)\right] \leqslant c, \omega \in A_{k}(\lambda) \cap D(c) .
$$

当 $\lambda>1$ 时将 (21) 式两边同除以 $\log \lambda$ 得

$$
\begin{gathered}
\left.\limsup _{n \rightarrow \infty}\left\{\frac{1}{n} S_{*}(k, \omega)-\sum_{i=1}^{\infty} p_{i k}\right]-\frac{1}{n} \sum_{i=1}^{*}\left[\frac{\log \left(1+(\lambda-1) p_{i k}\right)}{\log \lambda}-p_{i k}\right]\right\} \leqslant \frac{c}{\log \lambda}, \\
\omega \in A_{k}(\lambda) \cap D(c) .
\end{gathered}
$$

由 (22) 式及不等式 $1-\frac{1}{x} \leqslant \log x \leqslant x-1(x>0)$ 及 $\sum_{i=1}^{n} p_{i k} \leqslant n$ 得

$$
\begin{aligned}
\limsup _{n \rightarrow \infty} & \frac{1}{n}\left[S_{n}(k, \omega)-\sum_{i=1}^{n} p_{i k}\right] \\
& \leqslant \limsup _{n \rightarrow \infty} \frac{1}{n} \sum_{i=1}^{\infty}\left[\frac{\log \left(1+(\lambda-1) p_{i k}\right)}{\log \lambda}-p_{i k}\right]+\frac{c}{\operatorname{lng} \lambda} \\
& \leqslant \limsup _{i \rightarrow \infty} \frac{1}{n} \sum_{i=1}^{\infty}\left[\frac{(\lambda-1) p_{i k}}{1-1 / \lambda}-p_{i k}\right]+\frac{1}{1-1 / \lambda} \\
& \leqslant \lambda-1+c+\frac{c}{\lambda-1}, \omega \in A_{k}(\lambda) \cap D(c) .
\end{aligned}
$$

易知当 $c>0$ 时 $z(\lambda)=\lambda-1+c+\frac{c}{\lambda-1}(\lambda>1)$ 在 $\lambda=1+\sqrt{c}$ 处取到最小值 $g(1+\sqrt{c})=2 \sqrt{c}+c$, 于是由 (23) 式有

$$
\limsup _{n \rightarrow \infty} \frac{1}{n}\left[S_{n}(k, \omega)-\sum_{i=1}^{\infty} p_{i k}\right] \leqslant 2 \sqrt{c}+c, \omega \in A_{k}(1+\sqrt{c}) \cap D(c) \text {. }
$$


由于 $P\left(A_{k}(1+\sqrt{c})\right)=1$, 故由 (24) 式知当 $c>0$ 时 (7) 式成立. 当 $c=0$ 时, 取 $\lambda_{i}>1$ $(i-1,2, \cdots)$ 使 $\lambda_{i} \rightarrow 1+0(i \rightarrow \infty)$. 令 $H^{*}(k)=\bigcap_{i=1}^{\infty}\left(A_{k}\left(\lambda_{i}\right) \cap D(c)\right)$, 则对一切 $i$ 由 (23) 式有

$$
\underset{* \rightarrow \infty}{\limsup } \frac{1}{n}\left[S_{n}(k, \omega)-\sum_{i=1}^{n} p_{i k}\right] \leqslant \lambda_{i}-1, \omega \in H^{*}(k) .
$$

由于 $\lambda_{i}-1 \rightarrow 0(i \rightarrow \infty)$, 故由 (25) 式有

$$
\limsup _{n \rightarrow \infty} \frac{1}{n}\left[S_{n}(k, \omega)-\sum_{i=1}^{n} p_{i k}\right] \leqslant 0, \omega \in H^{*}(k) \text {. }
$$

由于 $H^{*}(k) \subset D(0)$ 且 $P\left(H^{*}(k)\right)=P(D(0))$, 故由 (26) 式知当 $c=0$ 时 (7) 式亦成立.

类似,利用当 $0<\lambda<1$ 时的 (21) 式可以证明当 $0 \leqslant c<1$ 时 (8) 式成立.

推论 $\lim _{n \rightarrow \infty} \frac{1}{n}\left[S_{*}(k, \omega)-\sum_{i=1}^{*} p_{i k}\right]=0$ a.e., $\omega \in D(0)$.

注. 在 (18) 式中取 $\lambda=1$ 得 $t_{*}(1, \omega)=\gamma_{n}(\omega)$, 于是由 (12) 与 (15) 式有

$$
\limsup _{n \rightarrow \infty}(\omega) \leqslant 0 \text { a.e., } \omega \in[0,1) \text {. }
$$

\section{参省文献}

[1] Barron, A. R., Ann. Prabab, 13(1986), 1292-1303.

[2] Shannon, C., Bell. Syszem. Techn. Journ, 27(1948), 379-423, 623-656.

[ 3 ] McMillan, B., Ann. Mazh. Stat, 24(1953), 169-219.

[4] Feinstein, A., IEE Trans. PGIT, 1954, 2-22.

[ 5 ] Breiman, L., Ann. Mazh. Staz, 28(1957), 809-811.

[ 6 ] Chung, K. L., Ann. Math. Stazst, 32(1961), 612-614.

[ 7 ] Kiefer, J. C., Pacific J. of Math., 51(1974), 203-204.

[8] 刘文, 应用概率统计, 4(1988),3: $287 \ldots 294$.

[9] Hildebrandt, T. H., Introduction to the Theory of Integration, Academic Press, New York, 1963. 